\title{
Commentary
}

\section{$n-3$ Fatty acids and mood: the devil is in the detail}

Increasing evidence suggests that a low dietary intake of the n-3 long-chain PUFA EPA and DHA may contribute not only to the risks for various physical illnesses (particularly cardiovascular and immune system disorders), but also to many disorders of mental health and performance ${ }^{1,2}$. From their recent comprehensive review of the evidence for these $n-3$ fatty acids in the prevention and treatment of psychiatric disorders $^{3}$, the American Psychiatric Association (APA) made the following recommendations:

- all adults should eat fish at least twice weekly;

- patients with mood, impulse control or psychotic disorders should consume $1 \mathrm{~g} / \mathrm{d}$ EPA + DHA (the $n$-3 fatty acids found in oily fish and fish oils);

- a supplement (providing 1-9g/d EPA + DHA) may be useful in patients with mood disorders. Use of $>3 \mathrm{~g} / \mathrm{d}$ should be monitored by a physician.

It was strongly emphasised that these recommendations are not intended as a substitute for standard treatments for psychiatric disorders, as most trials to date have used $n-3$ fatty acids adjunctively.

The recommendation of the APA of $1-9 \mathrm{~g} / \mathrm{d}$ EPA + DHA (combined) for mood disorders followed from their meta-analysis of eight trials involving adults with clinical depression or bipolar disorder, showing overall benefits from supplementation with these $n-3$ fatty acids $(P<0 \cdot 03)$. This showed considerable heterogeneity, however, so the need to examine carefully the differences between study populations, as well as specific formulations and doses used, was emphasised. It was also pointed out that treatment primarily or exclusively with EPA appeared to be most effective.

In view of this, it seems unfortunate that a high-DHA formulation was chosen for the trial reported in this issue of the $B J N$ by Rogers and colleagues ${ }^{4}$, which showed no effects on mood or cognition in generally healthy adults from 12 weeks of supplementation with $n-3$ fatty acids. Clearly this population differs from those considered by the APA, both in having no formal psychiatric diagnoses and in receiving no other treatment for their mood symptoms, but the rationale for the study was sensible. The prevalence of mild to moderate depression in primary care settings (where most participants were recruited) is high, and concerns about antidepressant medications, along with mounting evidence linking low $n-3$ fatty acid intake to mood disorders, have raised the very important question of whether simple dietary interventions to improve that intake might improve depressive symptoms in this population. The issues involved are not simple, however, and definitive answers must still await further research.
The evidence supporting the use of EPA + DHA in the management of psychiatric disorders appears strongest for conditions involving disturbances of mood/anxiety and/or impulse control. Thus in addition to the benefits for major depression and bipolar disorder highlighted by the APA's meta-analysis of studies of these patients ${ }^{3}, 1 \mathrm{~g} / \mathrm{d} \mathrm{EPA}$ reduced both depression and hostility in patients with borderline personality disorder ${ }^{5}$. In two newer studies, $1.2 \mathrm{~g}$ EPA $+0.9 \mathrm{~g}$ DHA improved depression, suicidality and daily stresses (but not impulsivity, aggression and hostility) in patients with recurrent self-harm ${ }^{6}$, and $2.3 \mathrm{~g} \mathrm{EPA}+0.5 \mathrm{~g}$ DHA reduced anxiety in a pilot study of substance abusers ${ }^{7}$. By contrast, a separate meta-analysis by the APA's reviewers of four trials involving schizophrenia patients showed no overall benefits for psychotic symptoms, although it was noted that an increased intake of $n$-3 fatty acids might help to combat the increased risks of CVD, diabetes and other physical health problems associated with this illness, which are exacerbated by some antipsychotic medications. Some benefits for mood, impulsivity, stress-aggression and other aspects of behaviour or cognition have also been reported in psychiatrically normal populations ${ }^{8,9}$, children with attention-deficit hyperactivity disorder and related conditions ${ }^{10}$, and patients with mild dementia ${ }^{11}$, although findings in these areas remain preliminary or mixed, and most studies have been small.

This new study by Rogers and colleagues ${ }^{4}$ set out to investigate whether $n-3$ fatty acid supplementation could improve mental health in a sample more representative of the general population. As they noted, existing evidence does suggest that adults with sub-clinical depressive symptoms might be likely candidates to benefit from an increased $n-3$ fatty acid intake. So their choice of a new population to study makes good sense. But pooling their results with those of other trials involving very different populations does not. This allencompassing approach to meta-analysis was used in an earlier publication from the same group ${ }^{12}$, and it is repeated in the current paper, with the inclusion of this latest trial. What have males with angina, chronic patients with schizophrenia, and mothers who choose to breastfeed got in common? They all show no improvements in 'mood' following ' $n-3$ fatty acid supplementation' in these meta-analyses. And what might ' $n-3$ fatty acid supplementation' mean here? Well, these chronic schizophrenia patients received $3 \mathrm{~g} / \mathrm{d}$ pure ethyl-EPA (other studies of schizophrenia using lower doses were not included), the breastfeeding mothers received only $200 \mathrm{mg} / \mathrm{d}$ pure algal-source DHA; and the angina sufferers were apparently just told to 'eat more fatty fish' or were given 'EPA capsules', with no dose reported. It seems less than meaningful to combine results from studies using 
such totally diverse populations and treatments. Furthermore, these three particular (negative) trials receive $50 \%$ of the total weight in the latest meta-analysis, and the new (negative) trial of generally healthy adults adds a further $17 \%$. The overall conclusion is therefore that EPA + DHA has no effect on mood, although the positive results in patients with diagnosed mood disorders are acknowledged elsewhere in both papers.

Useful meta-analyses require expertise in both methodology and the specific subject area. Combining different populations is one potential confound; but combining totally different treatment formulations and dosages can be equally misleading, and doing both together can multiply any confusion. The actual treatment used is obviously rather a key factor, but in this particular field it is all too often overlooked. Ideally, separate meta-analyses should be performed by formulation and dosage. Unfortunately, treatments have differed so widely in trials of $n-3$ fatty acids for mental health and performance that this approach remains impractical until more studies are forthcoming. As the APA group emphasised, close examination of individual trials is warranted in the meanwhile; and the current pattern shows a clear superiority of EPA over DHA for these purposes.

This is not what most people might have predicted, because DHA is an essential component of neuronal membranes and thus critical to the very structure of the brain and nervous system, while EPA is not. EPA can affect brain function, however, in a huge number of ways. Its eicosanoid derivatives are key regulators of immune, endocrine and cardiovascular functions, and EPA has direct actions on cyclo-oxygenases, lipoxygenases, phospholipases, acylating systems, ion channels, mitochondria and PPAR, as well as regulatory influences on gene expression. The mechanisms underlying any treatment benefits observed still remain a matter for speculation and future research, but so far EPA does seem to produce better results than DHA for adult psychiatric disorders.

Thus studies using either pure DHA, or more DHA than EPA, for adult mental health symptoms have all been negative to date; and Rogers and colleagues' study has joined this list. By contrast, pure EPA has shown benefits in several trials (but only at doses of $1-2 \mathrm{~g} / \mathrm{d}$ ), and all positive studies in this area have used more EPA than DHA (ratios 1.8 to infinity; most ordinary fish oils provide around 1.5). The few controlled trials in children with behavioural and learning difficulties also suggest that EPA may be more effective than DHA, although the picture here is less clear, and is complicated by other ingredients such as antioxidants and $n-6$ fatty acids ${ }^{10}$. There are just two underpowered dose-ranging trials to date, both in adults with psychiatric disorders, and using 1,2 or $4 \mathrm{~g} / \mathrm{d}$ pure ethyl-EPA. For depression, the $1 \mathrm{~g}$ dose performed best ( 2 or $4 \mathrm{~g}$ were no better than placebo $)^{13}$ and for schizophrenia, only the $2 \mathrm{~g}$ dose showed any benefits ${ }^{14}$. Any successful trials using higher doses have provided EPA and DHA in combination, and this may be important, as high doses of any one fatty acid could potentially create imbalances in fatty acid profiles.

In keeping with UK guidelines, Rogers and colleagues used $n-3$ fatty acid dose that was realistically achievable from the diet, in this case $1.5 \mathrm{~g} \mathrm{EPA}+$ DHA in total. This is above the $500 \mathrm{mg} / \mathrm{d}$ EPA + DHA recommended for maintaining cardiovascular health ${ }^{15}$. However, for reasons unspecified, an unusually low ratio of EPA:DHA of 0.7 was chosen and so the supplement provided less than the minimum $1 \mathrm{~g} / \mathrm{d}$ EPA indicated as efficacious by previous research. For a relatively large-scale study that otherwise appears extremely welldesigned and conducted, this seems a very strange decision. The negative results certainly add to the case against DHArich formulations for mood. But they also represent a wasted opportunity to find out if the existing evidence that $n-3$ fatty acids can (adjunctively) help patients with mood disorders might extend (as monotherapy) into the general population. It can only be hoped that neither this study, nor the use of over-inclusive meta-analyses, will deter the further research that is still urgently needed to address this question.

\section{Acknowledgements}

Financial support from the UK charity Food and Behaviour Research is gratefully acknowledged. Independently, I also act as a consultant to several companies that make foods or supplements containing fatty acids.

Alex J. Richardson

Department of Physiology Anatomy and Genetics

University of Oxford

Sherrington Building

Parks Road

Oxford OX1 3PT

$U K$

Email: alex.richardson@dpag.ox.ac.uk

\section{References}

1. Peet M, Glen I \& Horrobin DF (editors) (2006) Phospholipid Spectrum Disorders in Psychiatry and Neurology. Carnforth: Marius Press.

2. Vaddadi K (editor) (2006) Essential fatty acids and mental illness. In Int Rev Psychiatry, pp. 18, 81-186, Special Issue.

3. Freeman MP, Hibbeln JR, Wisner KL, Davis JM, Mischoulon D, Peet M, Keck PE Jr, Marangell LB, Richardson AJ, Lake J \& Stoll AL (2006) Omega-3 fatty acids: evidence basis for treatment and future research in psychiatry. J Clin Psychiatry 67, 1954-1967.

4. Rogers PJ, Appleton KM, Kessler D, Peters TJ, Gunnell D, Hayward RC, Heatherley SV, Christian LM, McNaughton SA \& Ness AR (2007) No effect of $n$-3 long-chain polyunsaturated fatty acid (EPA and DHA) supplementation on depressed mood and cognitive function: a randomised controlled trial. Br J Nutr 99, 421-431.

5. Zanarini MC \& Frankenburg FR (2003) Omega-3 fatty acid treatment of women with borderline personality disorder: a double-blind, placebo-controlled pilot study. Am J Psychiatry 160, 167-169.

6. Hallahan B, Hibbeln JR, Davis JM \& Garland MR (2007) Omega-3 fatty acid supplementation in patients with recurrent self-harm: single-centre double-blind randomised controlled trial. Br J Psychiatry 190, 118-122.

7. Buydens-Branchey L \& Branchey M (2006) n-3 Polyunsaturated fatty acids decrease anxiety feelings in a population of substance abusers. J Clin Psychopharmacol 26, 661-665.

8. Hamazaki T, Sawazaki S, Itomura M, Asaoka E, Nagao Y, Nishimura N, Yazawa K, Kuwamori $\mathrm{T}$ \& Kobayashi $\mathrm{M}$ (1996) The effect of docosahexaenoic acid on aggression in 
young adults. A placebo-controlled double-blind study. J Clin Invest 97, 1129-1133.

9. Fontani G, Corradeschi F, Felici A, Alfatti F, Migliorini S \& Lodi L (2005) Cognitive and physiological effects of Omega3 polyunsaturated fatty acid supplementation in healthy subjects. Eur J Clin Invest 35, 691-699.

10. Richardson AJ (2006) Omega-3 fatty acids in ADHD and related neurodevelopmental disorders. Int Rev Psychiatry 18, $155-172$.

11. Freund-Levi Y, Eriksdotter-Jonhagen M, Cederholm T, Basun H, Faxen-Irving G, Garlind A, Vedin I, Vessby B, Wahlund LO \& Palmblad J (2006) Omega-3 fatty acid treatment in 174 patients with mild to moderate Alzheimer disease: OmegAD study: a randomized double-blind trial. Arch Neurol 63, $1402-1408$.
12. Appleton KM, Hayward RC, Gunnell D, Peters TJ, Rogers PJ, Kessler D \& Ness AR (2006) Effects of n-3 long-chain polyunsaturated fatty acids on depressed mood: systematic review of published trials. Am J Clin Nutr 84, 1308-1316.

13. Peet M \& Horrobin DFA (2002) dose-ranging study of the effects of ethyl-eicosapentaenoate in patients with ongoing depression despite apparently adequate treatment with standard drugs. Arch Gen Psychiatry 59, 913-919.

14. Peet M \& Horrobin DFE-E-Multicentre-Study-Group (2002) A dose-ranging exploratory study of the effects of ethyl-eicosapentaenoate in patients with persistent schizophrenic symptoms. J Psychiatry Res 36, 7-18.

15. Recommendations for intake of polyunsaturated fatty acids in healthy adults ISSFAL Policy Statement 3, June Meeting, Brighton (www.issfal.org.uk/) 